\title{
Tractor accelerated test on test rig
}

\author{
M. Mattetti, ${ }^{1}$ Giovanni Molari, ${ }^{1}$ A. Vertua, ${ }^{2}$ A. Guarnieri ${ }^{1}$ \\ Dipartimento di Scienze e Tecnologie Agro-alimentari, Università di Bologna, Bologna, Italy
}

\begin{abstract}
The experimental tests performed to validate a tractor prototype before its production, need a substantial financial and time commitment. The tests could be reduced using accelerated tests able to reproduce on the structural part of the tractor, the same damage produced on the tractor during real life in a reduced time. These tests were usually performed reproducing a particular harsh condition a defined number of times, as for example using a bumpy road on track to carry out the test in any weather condition. Using these procedures the loads applied on the tractor structure are different with respect to those obtained during the real use, with the risk to apply loads hard to find in reality. Recently it has been demonstrated how, using the methodologies designed for cars, it is possible to also expedite the structural tests for tractors. In particular, automotive proving grounds were recently successfully used with tractors to perform accelerated structural tests able to reproduce the real use of the machine with an acceleration factor higher than that obtained with the traditional methods. However, the acceleration factor obtained with a tractor on proving grounds is in any case reduced due to the reduced speed of the tractors with respect to cars. In this context, the goal of the paper is to show the development of a methodology to perform an accelerated structural test on a medium power tractor using a 4 post test rig. In particular, several proving ground testing conditions have been performed to measure the loads on the tractor. The loads obtained were then edited to remove the not damaging portion of signals, and finally the loads obtained were reproduced in a 4 post test rig. The methodology proposed could be a valid alternative to the use of a proving ground to reproduce accelerated structural tests on tractors.
\end{abstract}

Correspondence: Giovanni Molari, Dipartimento di Scienze e Tecnologie Agro-alimentari, Università di Bologna, viale Fanin 50, Bologna Italy.

Tel. +39.051.2096191 - Fax: +39.051.2096178.

E-mail: giovanni.molari@unibo.it

Key words: durability, accelerated test, tractor, 4-post rig.

CCopyright M. Mattetti et al., 2013

Licensee PAGEPress, Italy

Journal of Agricultural Engineering 2013; XLIV(s2):e76

doi:10.4081/jae.2013.s2.e76

This article is distributed under the terms of the Creative Commons Attribution Noncommercial License (by-nc 3.0) which permits any noncommercial use, distribution, and reproduction in any medium, provided the original author(s) and source are credited.

\section{Introduction}

Recently the competitiveness between different tractor manufacturers has increased in particular with reference to the increasing of the reliability (Strutt and Hall, 2003) and to the reduction of the time-tomarket similarly to the automotive trend (Hughes et al., 2005). One of the development steps more involved in this tendency is the durability approval due to the high time-consumption (Oelmann, 2002). This development phase consists of an application of a load sequence, representative of the real use of the machine, to the whole vehicle or to a specific component (Garcia et al., 2010). The load sequence reproduces in the structure a damage equivalent to that obtained by the farmer's use in the whole life of the tractor. A detailed measure of the real loads on a similar machine during the customer usage, a removal of the not damaging cycles (Lee et al., 2005), and the reproduction of the load cycle obtained on a tractor prototype are therefore necessary.

The reproduction of the load cycle on a real field is difficult, not only due to the dependency on weather conditions, but also for the reduced occurrence of the applied loads and, as a consequence, a long time necessary to reproduce the entire cycle. To overcome these problems, particular tracks with defined bumps were designed, but despite a reduction of the test time they have not permitted an accurate reproduction of the load cycles (Mattetti et al., 2012). In the recent years different studies have been performed to improve the feasibility of these tests (Thomas et al., 1999; Renius, 1977; Kim et al, 2006; Kim et al., 2006). One of these methods involved the use of proving grounds, special tracks with different types of pavements that are possible to meet during the real car driving (Ensor and Cook, 2007). The use of proving grounds permits a

reduction of the testing time due to a high occurrence of the stress and due to the high occurrence of higher damaging events with respect to the field. The use of the proving grounds have permitted to reproduce a load cycle of a medium power tractor with an acceleration factor of about three (Mattetti et al., 2012). This acceleration factor is around three times, lower with respect to the usual one obtained for cars (Braccesi et al., 2005), due to the lower speed of the tractor and the difficulties in the reproduction of the horizontal loads. Other problems related to the use of the proving grounds are the weather conditions and the necessity to change the drive after some hours. To further increase the acceleration factor of the tests a 4 post test rig could be used. This rig is provided by hydraulic cylinders, transducers and control systems used to replay the load cycle on the vehicle. The reproduction of the load cycle on the 4 post test rig could reduce the time of the proving ground tests due to the possibility to perform these tests in the lab without a driver on the tractor (Ledesma et al., 2005). The goal of the paper is the definition of a methodology to reproduce the load cycles necessary for the durability approval also in a 4 post test rig with an acceleration factor higher with respect to that obtained with only proving grounds. 
Table 1. Measured channels list

\begin{tabular}{lcc} 
Channel & Measured channel & Hand \\
1 & Lift arm load & Left \\
2 & Lift arm load & Right \\
\hline 3 & Top link load & - \\
4 & Lower arm axial load & Left \\
\hline 5 & Lower arm axial load & Right \\
6 & Vertical front axle load & Left \\
\hline 7 & Vertical front axle load & Right \\
8 & Vertical front axle acceleration & Left \\
\hline 9 & Vertical front axle acceleration & Right \\
10 & Vertical rear axle acceleration & Left \\
\hline 11 & Vertical rear axle acceleration & Right \\
\hline
\end{tabular}

\section{Materials and methods}

The methodology was applied to a $80 \mathrm{~kW}$ power tractor. In the tractor the transducers indicated on Table 1 were fitted to measure the load on the lift arm, on the top link, on the lower arm, on the vertical front axle; and the accelerations on the vertical front and rear axle.

The test schedule has been defined in Mattetti et al., (2012) to reproduce the loading cycle derived from the signals obtained during tests on field. In particular the test schedule was composed of a combination of different proving grounds (PG) travelled a defined number of times and two field operations (F0), also repeated a defined number of times. The signals correspondent to the channels 1-7 reported on Table 1 were edited to reproduce a part of the test schedule on the 4 post test rig. The editing was obtained using the software nCode GlyphworksTM (). In particular the editing was realised splitting the signals into temporal windows in which the pseduo-damage was calculated according to:

$$
P D=\sum_{i} n_{i} S_{i}^{4}
$$

where:

$\mathrm{S}_{\mathrm{i}}$ : load amplitude derived from rainflow matrix;

$\mathrm{n}_{\mathrm{i}}$ : cycle number counted in a generic time history;

with $S_{\mathrm{i}}$ and $\mathrm{n}_{\mathrm{i}}$ calculated using the rainflow algorithm (Downing and Socie, 1982; Rychlik, 1987).

The signals were edited removing the less damaging portions, and maintaining a damage higher than $95 \%$ of the total damage. The removal of some portions of the signal introduces unwanted spikes that modify the frequency content of the signal and could damage the rig. For this reason the not continuous windows were connected with halfsine joining functions. Once defined the windows of the force signals, the correspondent windows of the acceleration signals were composed to be reproduced in the 4 post test rig. From the acceleration the drives of the hydraulic cylinders of the 4 post test rig were obtained to reproduce the load cycle on the different channels (Kelly et al., 2002).

\section{Results}

The signals of one test condition with the deleted parts highlighted are reported in Figure 1. The deleted parts of the signals are in preponderance in the starting and final parts of the signals.
The edited signals have a similar trend with respect to the measured ones with the only difference being the duration of the signal. In Figure 3 the power spectrum densities are compared, in particular the two trends are very similar due to the fact that the editing does not introduce anomalous peaks.

The tractor could be tested on the 4 post test rig for a reduced duration with respect to the different tests on proving grounds (PG) and on

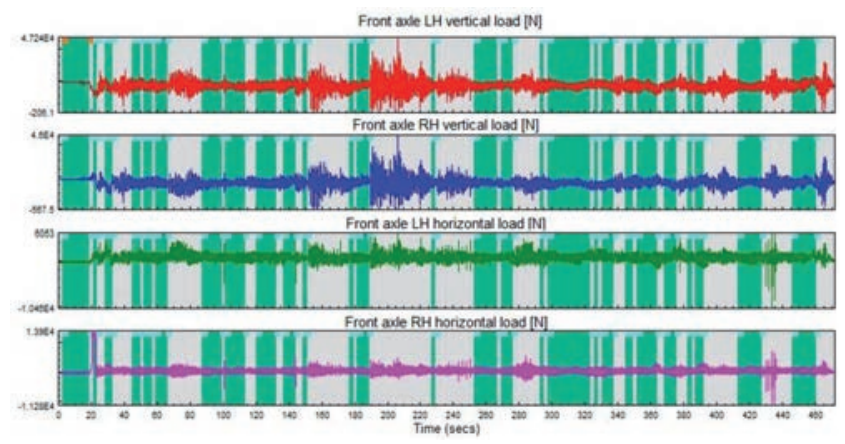

Figure 1. Editing of the signals: the green parts are the not damaging part of the signals.

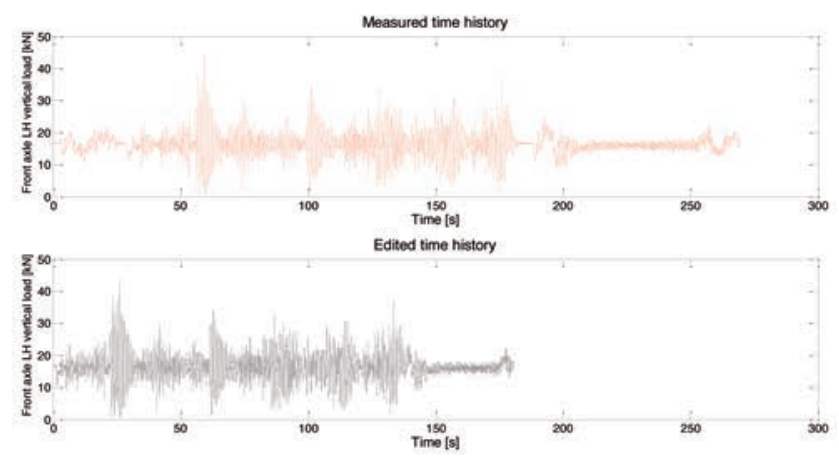

Figure 1. Editing of the signals: the green parts are the not damaging part of the signals.

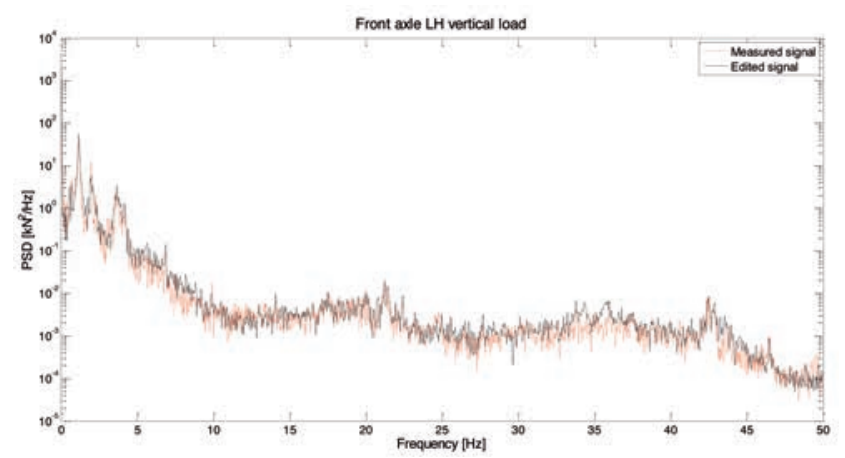

Figure 3. Comparison between the Power Spectrum Density of the measured signal and the edited one. 
Table 2. Reduction factor for each test condition.

\begin{tabular}{lcc} 
Testing condition & Duration of the tests withoutwith the combination with the test on $\mathbf{4}$ post test rig [s] & Reduction factor [\%] \\
PG test condition 1 & 181 & 33 \\
PG test condition 2 & 370 & 41 \\
\hline PG test condition 3 & 373 & 11 \\
PG test condition 4 & 368 & 9 \\
\hline PG test condition 5 & $\mathbf{3 7 0}$ & $\mathbf{1 5}$ \\
\hline PG test condition 6 & 42 & 69 \\
\hline FO test condition 1 & 49 & 78 \\
FO test condition 2 & & \\
\hline
\end{tabular}

field (F0). The reduction factor for each test condition with respect to the time necessary on PG and on F0 are reported on Table 2.

Testing condition Duration of the tests without/with the combination with the test on 4 post test rig [s] Reduction factor [\%] PG test condition 118133 PG test condition 227041 PG test condition 337311 PG test condition 43739 PG test condition 53688 PG test condition 6370 15 FO test condition 14269 FO test condition 24978

The two field operations are the activities with the highest reduction coefficients due the higher portion of not damaging signals. But the two field operations are the activities with the higher influence on the total time of the test. The using of the 4 post test rig permits a reduction of the total time of the test into only 500 hours instead of the 1100 hours obtained by Mattetti et al. (2012) with the use of PG and FO only.

Furthermore the test on the 4 post test rig can be performed without interruption due to the change of the driver or unfavourable weather conditions as for the proving ground and field work tests.

\section{Conclusions}

In the paper a methodology to perform accelerated structural tests on a tractor has been presented. In particular, starting from a test schedule composed of a combination of proving grounds and field works, tests on a 4 post test rig have been performed to reduce the total time of the test. The use of the 4 post test rig permits a reduction of the total time of the test in only 500 hours instead of the 1100 hours obtained with the use only of proving grounds and field works. The total acceleration factor with the 4 post test rig was about 6 .

\section{References}

Braccesi C, Cianetti F, Pioli D. Optimisation of the process of experimental sign off of a vehicle. Int J Heavy Veh Syst 2005;12:193-206.

Downing SD, Socie D. Simple rainflow counting algorithms. Int J Fatigue $1982 ; 4: 31-40$.

Ensor D, Cook C. Derivation of Durability Targets and Procedures Based on Real World Usage. SAE Technical Paper 2007; 2007-26-074.

Garcia C, Araan J, Ruiz S. Design of reliable accelerated test programs based on real market use 2010; 2010-36-0029.

Hughes S, Jones RP, Burrows AJ. Application of System Modeling to Road Load Data Synthesis for Automobile Product Development. In Proceedings of the ASME Computers and Information in Engineering Division ASME Conference Proceedings, 2005 Nov 511, Orlando, Florida. Michingan:ASME; 2005. pp31-40.

Kelly J, Kowalczyk H, Oral HA. Track Simulation and Vehicle Characterization with 7 Post Testing. SAE Technical Paper 2002; 2002-01-3307.

Kim, HE, Kim DS, Lee YP, Yoo YC. Accelerated Life Testing Method of Transmission. Key Eng Mat 2006; 326-8:1865-8.

Ledesma R, Jenaway L, Wang Y, Shih S. Development of Accelerated Durability Tests for Commercial Vehicle Suspension Components. SAE Technical Paper 2005; 2005-01-3565.

Lee YL, Pan J, Hathaway RB, Berkley ME. Fatigue testing and analysis: theory and practice. Burlington, MA: Butterworth-Heinemann. Burlington;2005.

Mattetti M, Molari G, Sedoni E. Methodology for the realisation of accelerated structural tests on tractors. Biosyst Eng 2012; 113:266-71

Oelmann B. Determination of load spectra for durability approval of car drive lines. Fatigue Fract Eng M 2002; 25:1121-5.

Renius KT. (1977). Application of cumulative damage theory to agricultural tractor design elements. Konstruktion 1997; 29:85-93.

Rychlik I. A new definition of the rainflow cycle counting method. Int J Fatigue 1987; 9:119-21.

Strutt JE, Hall PL. Global vehicle reliability: prediction and optimization techniques. Suffok: Professional Engineering Pub; 2003.

Thomas JJ, Perroud G, Bignonnet A, Monnet D. Fatigue design and reliability in the automotive industry. Esis Publ 1999; 23:1-11 